\title{
Study on the Fluorescence Spectra Characteristics of Vinegar-Water Solutions
}

\author{
Dan Li, ${ }^{1,2}$ Xuan Wang, ${ }^{1,2}$ and Caiqin Han ${ }^{1,2}$ \\ ${ }^{1}$ School of Physics and Electronic Engineering, Jiangsu Normal University, Xuzhou 221116, China \\ ${ }^{2}$ Jiangsu Key Laboratory of Advanced Laser Materials and Devices, Jiangsu Normal University, Xuzhou 221116, China \\ Correspondence should be addressed to Caiqin Han; hancqin@163.com
}

Received 29 November 2015; Accepted 6 April 2016

Academic Editor: Maria Carmen Yebra-Biurrun

Copyright (c) 2016 Dan Li et al. This is an open access article distributed under the Creative Commons Attribution License, which permits unrestricted use, distribution, and reproduction in any medium, provided the original work is properly cited.

\begin{abstract}
Fluorescence spectra and polarization spectra of vinegar-water solutions with different concentration of $\mathrm{CH}_{3} \mathrm{COOH}$ have been studied. The characteristics and mechanism of fluorescence spectra are discussed, and polarization degree is calculated. Vinegarwater solutions are excited by ultraviolet (UV) light at $380 \mathrm{~nm}$. The characteristic fluorescence peaks of the solution were identified at $445 \mathrm{~nm}$ and $470 \mathrm{~nm}$. The type of emission fluorescence is $\pi^{*} \rightarrow \mathrm{n}$. With the increasing concentration of $\mathrm{CH}_{3} \mathrm{COOH} / \mathrm{pH}$ value, the peak intensity is enhanced first, and then fluorescence quenching occurs. The polarization degree confirms the molecular orientation of different sample solutions. This research provides theoretical and experimental basis for the physical/chemical properties and quality of vinegar detection by florescence spectroscopy.
\end{abstract}

\section{Introduction}

Although it is one of the most important sour fermented liquid seasonings, mature vinegar can be used for medicinal purposes. It is helpful to reduce the risks of hypertension, hepatitis, and skin disease. However, in recent years, excessive additives and industrial acetic acid are added into vinegar in order to lower the cost of production. It is known that ingesting excess additives or inedible industrial acetic acid would cause physical harm to the human body $[1,2]$. More seriously, long-term ingestion could cause major damage to health. Therefore, in order to ensure the quality of vinegar, studies of vinegar safety are necessary to be carried out. Numerous scientific researches on vinegar have been done in recent years [3-6]. With the combination of factor analysis of multiplex fluorescence in parallel, Callejón et al. classified different Shirley vinegar. Their method demonstrates a good way to extract relevant chemical information about the vinegars as well as classify and discriminate them considering the different ageing [3]. Wu et al. studied the biodiversity of lactic acid and acetic acid bacteria in the process of Shanxi mature vinegar's fermentation, and they found Acetobacter pasteurianus showing great variety in both genotypic and phenotypic tests [4]. Nagashima carried out comprehensive analysis on the antioxidant activity of a new type of black vinegar named "IZUMI." The results suggest that "IZUMI" increases antioxidant activity and reduces oxidative stress and blood filtration time in female subjects [5]. Wang et al. compared and determined the acetic acid of plum vinegar via visible/near infrared (Vis/NIR) spectroscopy and multivariate calibration; the results indicated that Vis/NIR spectroscopy combined with chemometrics could be used as an inexpensive and efficient way for the determination of acetic acid of plum vinegar [6]. All these studies have built a good foundation for the development of vinegar safety. However, there are a few quantitative researches on the fluorescence spectral characteristics and group structure of vinegar that have extensive application for scientific research and engineering practices of vinegar.

Fluorescence spectrometry is a detection method which is convenient, quick, and accurate. After excitation by ultraviolet or visible light, substances such as amidogen or hydroxyl would generate fluorescent light, which can reveal specific characteristics of the material. Fluorescence spectrometry can carry out the qualitative and quantitative analysis of the fluorescence. It is one of the most effective ways to study molecular structure and structural features [7]. For example, 
Guzmán et al. evaluated the quality of olive oil via fluorescence analysis, and the results presented demonstrate the ability of the fluorescence technique to characterize olive oils on the basis of all the quality parameters studied [8]. Maki et al. used perylene diimide and nitrogen oxides (PBILN) body as fluorescence reagent, with flow injection fluorescence method to measure ascorbic acid. The analytical results were in good agreement with those obtained using a conventional method [9]. Iizuka et al. performed fluorescence determination of melatonin by high-performance liquid chromatography (HPLC), and they obtained the structure of fluorescent products of melatonin [10]. Zhang et al. classified phytoplankton with a fluorescent method, and the sinusoidal-amplitudemodulation (SAM) technique was used in this method. Results revealed that this method has advantages of better detection limit and shorter detection time compared with the existing method [11]. Zhao and Li performed colorimetry and fluorescent detection of anionic surface active agent with water soluble sensors. The study found that the detection has the advantage of visual detection and high selectivity and sensitivity [12]. But so far, fluorescence spectrum analysis of vinegar has not attracted enough attention due to the complex ingredient of vinegar. Studies about fluorescence spectra of vinegar are rarely reported.

In this study, vinegar-water solutions emit fluorescence with ultraviolet excitation. The spectral properties and fluorescence changing rules of those vinegar-water solutions are studied. The relationship between fluorescence spectra of different samples and their molecular clusters is also explored. Their characteristics are explained with polarized spectra of $0^{\circ}$ and $90^{\circ}$. The experimental results provide references for future studies about physical/chemical properties and cluster structures of vinegar.

\section{Materials and Methods}

2.1. Sample Preparation. The experimental samples of vinegar solutions were produced by Hengshun Vinegar Industry Co., Ltd. The ingredients of vinegar are water, sorghum, barley, and peas. The vinegar was diluted with distilled (DI) water $(\rho>0.5 \mathrm{M} \Omega \cdot \mathrm{cm})$ into different samples with volume percentage of $1 \% \sim 10 \%$ (the step is $1 \%$ ). According to the label of vinegar, the concentration of the acid is $\sim 45 \mathrm{~g} / \mathrm{L}$; thus, the acetic acid concentrations of each sample were figured out in the range of $0.45 \sim 4.5 \mathrm{~g} / \mathrm{L}$ (the step is 0.45 ). Samples were stored at room temperature.

2.2. Experimental Apparatus and Method. A steady-state and time-resolved fluorescence spectrometer FLS920 (The Edinburgh Instruments Co., Ltd) was used in the experiment, wherein the light source power of the steady-state spectrum measurement is a Xenon lamp of $450 \mathrm{~W}$. The tunable wavelength range is $200 \sim 900 \mathrm{~nm}$, and there are Glan Thompson prism instruments with the polarizer and analyzer in the excitation incident light path and the fluorescence emission light path, respectively, which can be manually set angle and detection angle. The instrument's parameter settings were set as follows. The slit band width of excitation light was $3 \mathrm{~nm}$. The slit band width of emission light was $1 \mathrm{~nm}$. Correction

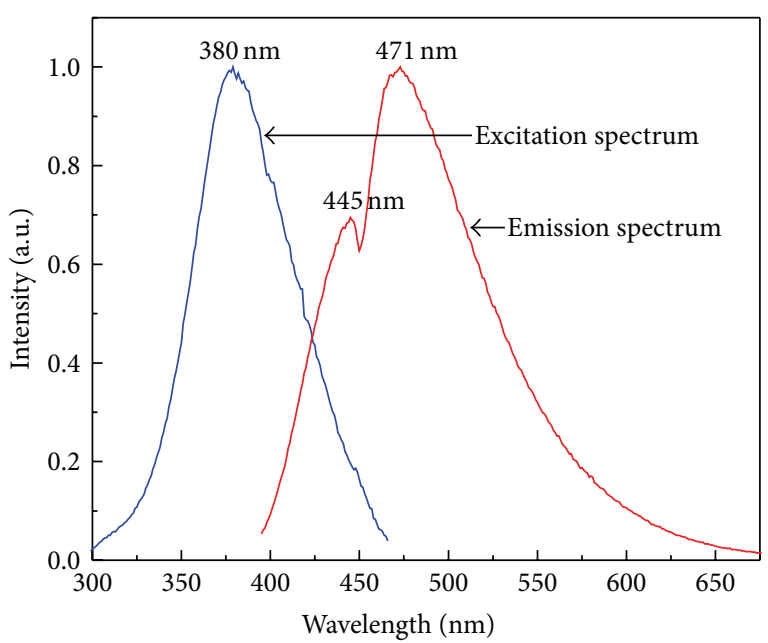

FIGURE 1: Normalized excitation spectrum and emission spectrum of vinegar-water solutions.

was used to eliminate the detector's error caused by the different time of different excitation light response. The scan time of each wavelength was $0.1 \mathrm{~s}$.

$1 \mathrm{~mL}$ as-prepared samples were added into the fluorescent quartz cuvettes with a size of $10 \mathrm{~mm} \times 10 \mathrm{~mm} \times 40 \mathrm{~mm}$. In order to ensure the comparability of the results, the parameters of fluorescence spectrum detecting of all samples were the same. The scanning range was $200 \sim 700 \mathrm{~nm}$, and the scan time of each wavelength was $0.1 \mathrm{~s}$. Samples were shaken to ensure homogeneity of the solution before each test. All fluorescence detections were performed at room temperature.

\section{Results and Discussion}

3.1. The Excitation Spectrum and Fluorescence Spectrum of Vinegar-Water Solutions. Figure 1 shows the excitation and emission spectra of vinegar-water solutions. It is found that light with wavelength of about $380 \mathrm{~nm}$ is absorbed by vinegarwater solutions. And fluorescence spectrum with a peak at $470 \mathrm{~nm}$ is emitted. In order to obtain the best excitation wavelength, multiband excitation spectrum scanning was performed with regard to the fluorescence peak at $470 \mathrm{~nm}$. The scanning range is $300 \sim 450 \mathrm{~nm}$, and scanning interval is $1 \mathrm{~nm}$. According to Figure 1, the maximum absorption appears at $380 \mathrm{~nm}$. Thus, the best excitation light wavelength for vinegar-water solutions is $380 \mathrm{~nm}$. The emission spectrum of vinegar-water solutions in Figure 1 is obtained by $380 \mathrm{~nm}$ UV light excitation. The peaks in $400 \sim 600 \mathrm{~nm}$ are the main fluorescence peaks of vinegar-aqueous solutions. The main peak is located at $471 \mathrm{~nm}$, and there is a weaker peak at $445 \mathrm{~nm}$.

According to the excitation and emission spectra of vinegar-water solutions in Figure 1, vinegar-water solutions can absorb photons of UV light at $380 \mathrm{~nm}$ and then emit fluorescence. It demonstrates that vinegar-water solutions contain the corresponding absorption structure. In addition to water, the major ingredient of vinegar is acetic acid $\left(\mathrm{CH}_{3} \mathrm{COOH}\right)$, which contains chromophores of carbonyl 
TABLE 1: The fluorescence intensities and intensities ratio with different concentrations of two peaks. Among them, "a.u." indicates arbitrary units.

\begin{tabular}{lcccccccccc}
\hline $\begin{array}{l}\text { Fluorescence } \\
\text { intensity/a.u. }\end{array}$ & 0.45 & 0.9 & 1.35 & 1.8 & 2.25 & 2.7 & 3.15 & 3.6 & 4.05 & 4.5 \\
\hline$I_{445}$ & 50230 & 71030 & 67850 & 60180 & 47520 & 40390 & 31530 & 25300 & 20660 & 14090 \\
$I_{470}$ & 62310 & 90130 & 91540 & 83460 & 59430 & 59570 & 49150 & 40910 & 33660 & 24020 \\
$I_{470} / I_{445}$ & 1.2405 & 1.2689 & 1.3492 & 1.3868 & 1.2506 & 1.4749 & 1.5588 & 1.6170 & 1.6292 & 1.7048 \\
\hline
\end{tabular}

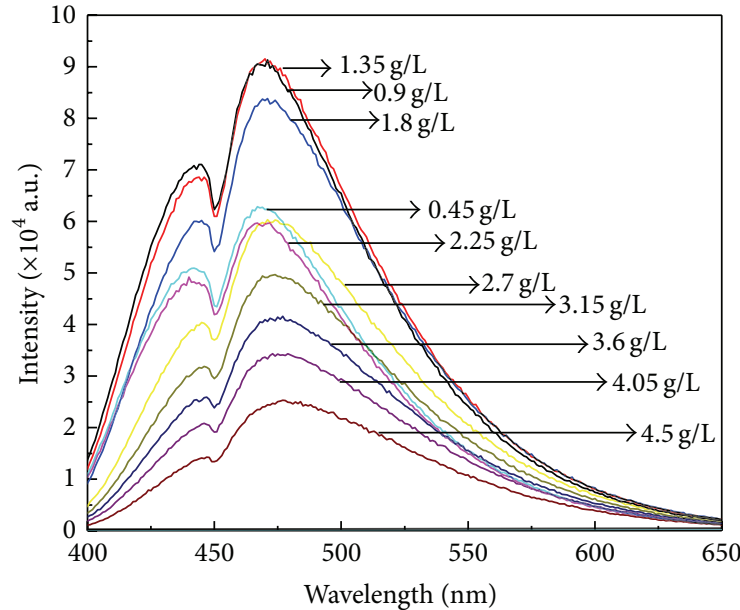

FIGURE 2: Fluorescence spectra of vinegar-water solutions with different $\mathrm{CH}_{3} \mathrm{COOH}$ concentrations.

$(\mathrm{C}=\mathrm{O})$ and hydroxyl $(-\mathrm{OH})$. It is known that organic molecules have four electron transition pathways in ultraviolet and visible light range. They are $\sigma \rightarrow \sigma^{*}, \mathrm{n} \rightarrow \sigma^{*}$, $\mathrm{n} \rightarrow \pi^{*}$, and $\pi \rightarrow \pi^{*}$. The energy requirement of each transition follows this relationship: $\sigma \rightarrow \sigma^{*}>\mathrm{n} \rightarrow \sigma^{*}>$ $\pi \rightarrow \pi^{*}>\mathrm{n} \rightarrow \pi^{*}$ [13]. The two electron transition ways including $\mathrm{n} \rightarrow \sigma^{*}$ and $\pi \rightarrow \pi^{*}$ generally absorb light that has a wavelength shorter than $250 \mathrm{~nm}$, while the light scanning range in this study is $350 \sim 700 \mathrm{~nm}$. Then, the transition of $\mathrm{n} \rightarrow$ $\pi^{*}$ is detected [14]. At the same time, electron (n) in hydroxyl jumps to the first excited electronic state by absorbing the energy of incident photon, which is the transition way of $\mathrm{n} \rightarrow$ $\pi^{*}$. The two peaks in Figure 1 represent the fluorescence peaks emitted by carbonyl and hydroxyl after absorbing corresponding photons. When an electron jumps from the lowest vibration energy level of excited singlet state back to the ground state, it may transition to many different vibrational and rotational energy levels on the same electron energy level; then, fluorescence with different wavelength is emitted [15]. On the other hand, the thermal radiation effect also affects the fluorescence wavelength; thus, a wide fluorescent band forms in the range of $400 \sim 600 \mathrm{~nm}$.

3.2. Fluorescence Spectra of Vinegar-Water Solutions Excited by Light at $380 \mathrm{~nm}$. Figure 2 shows the fluorescence spectra of vinegar-water solutions with different concentration of $\mathrm{CH}_{3} \mathrm{COOH}$ excited by UV light at $380 \mathrm{~nm}$. The fluorescent peaks of all samples are in the range of $400 \sim 600 \mathrm{~nm}$.
The shapes of fluorescence emission spectra are unchanged, and the main peaks are located at $\sim 470 \mathrm{~nm}$. With the increasing concentration of $\mathrm{CH}_{3} \mathrm{COOH}$ of the samples, the fluorescence intensity is gradually increasing until reaching a specific $\mathrm{CH}_{3} \mathrm{COOH}$ concentration value. When the concentration of $\mathrm{CH}_{3} \mathrm{COOH}$ reaches $1.35 \mathrm{~g} / \mathrm{L}$, the fluorescence intensity is maximized. However, the continuously increasing concentration of $\mathrm{CH}_{3} \mathrm{COOH}$ causes the declination of fluorescence intensity, which is concentration quenching effect. The intensity of minor peak exhibits the same changing trend as the main peak. According to Lambert Beer's law [16], when the concentration of a certain solution is very low, the relationship between absorbance and concentration of the solution follows the following equation:

$$
A=k d c,
$$

where $A$ is absorbance, $k$ is absorption coefficient, $d$ is optical path, and $c$ is concentration of the solution. The fluorescence emission intensity is proportional to $A$. Therefore, the fluorescence intensities increase when the $\mathrm{CH}_{3} \mathrm{COOH}$ concentrations increase at first. The fluorescence intensities decline when the concentration is higher than $1.35 \mathrm{~g} / \mathrm{L}$ because of fluorescence quenching effect. The fluorescence quenching effect is caused not only by the enhanced energy dissipation of nonradiation transition, but also by the increased probability of nonradiation transition with increasing concentration [17].

According to the experimental data from Figure 2, we obtain the intensity ratio of two peaks corresponding to each concentration. In Table 1, each $I_{470}$ is higher than the corresponding $I_{445} \cdot I_{470} / I_{445}$ slowly increases with the increasing $\mathrm{CH}_{3} \mathrm{COOH}$ concentration at the beginning; when the concentration increases to $2.25 \mathrm{~g} / \mathrm{L}, I_{470} / I_{445}$ decreases suddenly and continues to increase with the increasing concentration. First of all, sensitivities to the incident light of fluorescent chromophores corresponding to two peaks are different. Thus, the chromophores in solution are more likely to emit fluorescence under the irradiation with the wavelength of $470 \mathrm{~nm}$, and $I_{470}$ of any concentration is higher than the corresponding $I_{445}$. Secondly, in the early stages of the increasing $\mathrm{CH}_{3} \mathrm{COOH}$ concentration, the number of fluorescent chromophores in solution increases, which leads directly to the increasing of $I_{470}$; thus, $I_{470} / I_{445}$ is enhanced in the beginning. The decreasing of $I_{470} / I_{445}$ when the concentration increases to $2.25 \mathrm{~g} / \mathrm{L}$ is because the number of fluorescent chromophores increases to a certain amount and the collision probability increases, which promotes the nonradiation transition [18-20]. Therefore, $I_{470}$ decreases, resulting in suddenly declination of $I_{470} / I_{445}$. After that, $I_{470} / I_{445}$ continues 


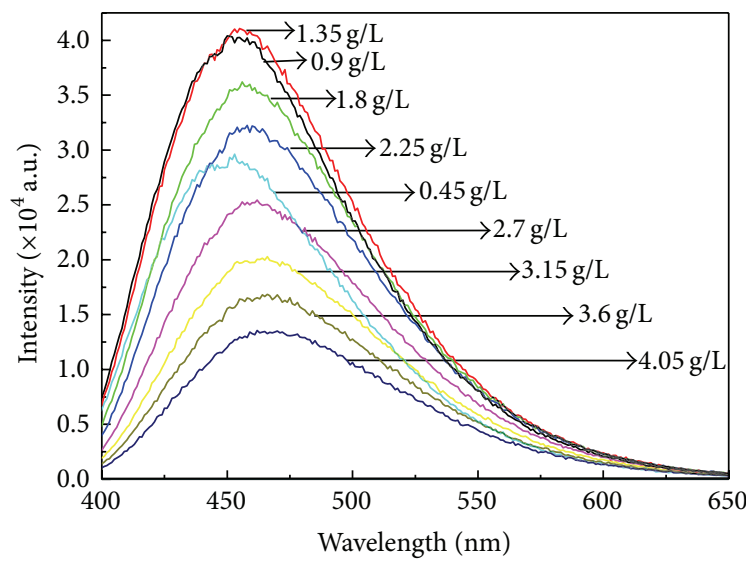

(a)

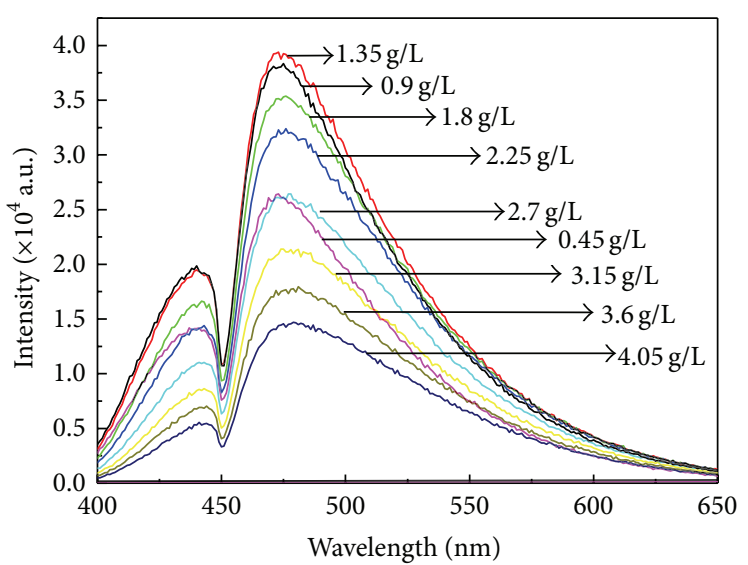

(b)

FIGURE 3: Polarization fluorescence spectra of vinegar-water solutions with different $\mathrm{CH}_{3} \mathrm{COOH}$ concentrations: (a) $0^{\circ}$ and (b) $90^{\circ}$.

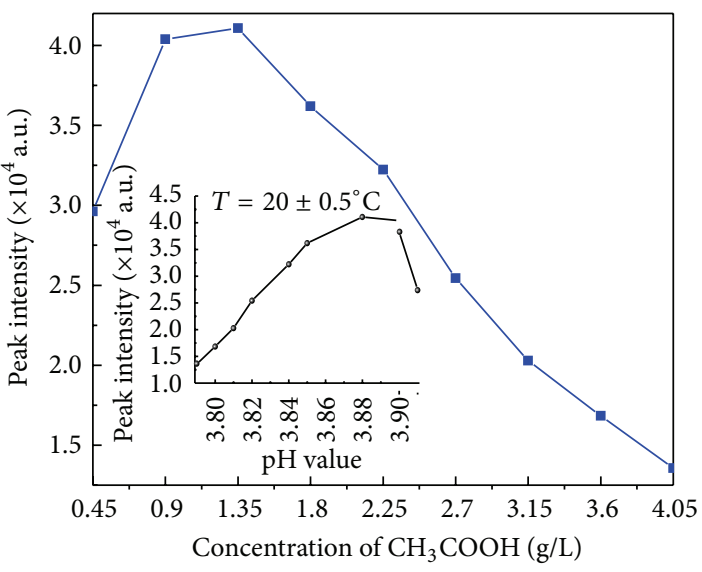

(a)

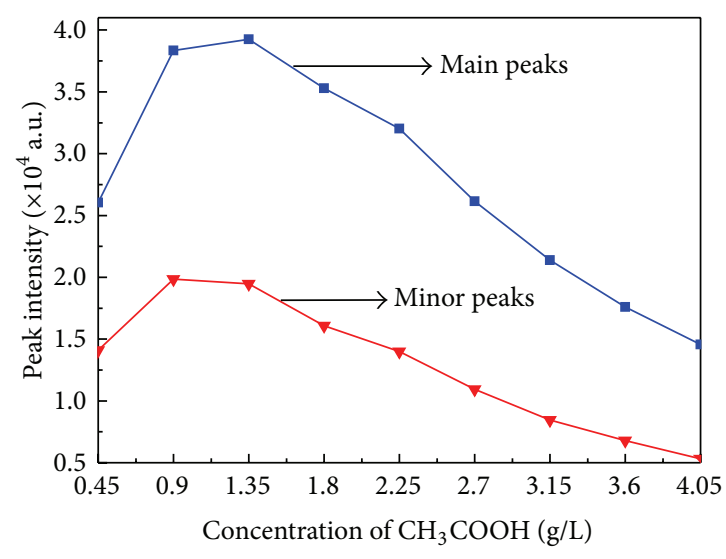

(b)

FIGURE 4: Line chart of polarized fluorescence peak intensities of vinegar-water solutions with different $\mathrm{CH}_{3} \mathrm{COOH}$ concentrations: (a) $0^{\circ}$ and (b) $90^{\circ}$.

to increase when $\mathrm{CH}_{3} \mathrm{COOH}$ concentration increases. The reason is that as the concentration increases, the number of fluorescent chromophores is still growing. At this time, energy loss resulting from collisions between molecules is weaker than fluorescence intensities emitted by lots of fluorescent chromophores. Obviously, the energy loss does not affect the overall enhancement trend of the fluorescence emission intensity; then, $I_{470} / I_{445}$ increases.

3.3. Polarization Fluorescence Spectra of Vinegar-Water Solutions. Figure 3 shows the polarization fluorescence spectra of vinegar-water solutions measured with inspection angles of $0^{\circ}$ and $90^{\circ}$. When the solutions are detected with the same inspection angle, the shape and peak positions of each sample have barely changed. The peak in Figure 3(a) detected with $0^{\circ}$ inspection angle reveals a peak at $\sim 457 \mathrm{~nm}$. Peaks in Figure 3(b) detected with $90^{\circ}$ inspection angle are located at $\sim 440 \mathrm{~nm}$ and $\sim 475 \mathrm{~nm}$. The results show that the fluorescence intensity gradually increases with the increasing concentration of $\mathrm{CH}_{3} \mathrm{COOH}$ at first. Then, when the concentration reaches a certain value, the fluorescence intensity maximizes. However, the concentration quenching effect appears when the concentration continues to increase. The relationship of peak intensity versus $\mathrm{CH}_{3} \mathrm{COOH}$ concentration is presented in Figures 4(a) and 4(b). The peak intensity differences between samples with $\mathrm{CH}_{3} \mathrm{COOH}$ concentrations of $0.9 \mathrm{~g} / \mathrm{L}$ and $1.35 \mathrm{~g} / \mathrm{L}$ are not obvious. However, the fluorescence peak intensity increases notably when $\mathrm{CH}_{3} \mathrm{COOH}$ concentration increases from 0.45 to $0.9 \mathrm{~g} / \mathrm{L}$. The peak intensity of other samples gradually decreases, but the attenuation amplitude is smaller than the enhancement. In addition, the relationship figure of peak intensity versus $\mathrm{pH}$ change is inserted in Figure 4(a), and the $\mathrm{pH}$ values are obtained by measuring vinegar solutions with different $\mathrm{CH}_{3} \mathrm{COOH}$ concentrations ranging from 0.45 to $4.05 \mathrm{~g} / \mathrm{L}$. Obviously, fluorescence intensity gradually increases with the increasing $\mathrm{pH}$ value and reaches a maximum value when the $\mathrm{pH}$ value is 3.88 . When the $\mathrm{pH}$ value reduces, the fluorescence quenching is caused by the proton of the acid group. On the other hand, the coordination ability of the hydrogen bond is strengthened and the solubility 
TABLE 2: The fluorescence peak intensities of vinegar-water solutions with the polarization angle of $0^{\circ}$ and $90^{\circ}$.

\begin{tabular}{lcccccccccc}
\hline $\begin{array}{l}\text { Fluorescence } \\
\text { intensity/a.u. }\end{array}$ & $\begin{array}{c}\text { Polarization } \\
\text { angle/ }^{\circ}\end{array}$ & 0.45 & 0.9 & 1.35 & 1.8 & 2.25 & 2.7 & 3.15 & 3.6 & 4.05 \\
\hline \multirow{2}{*}{$I_{440}$} & 0 & 28030 & 37620 & 37360 & 31400 & 27310 & 20920 & 15900 & 13080 & 9942 \\
& 90 & 14110 & 19850 & 19480 & 16080 & 14000 & 10940 & 8462 & 6792 & 5317 \\
$I_{475}$ & 0 & 24270 & 34760 & 36040 & 32630 & 29810 & 23910 & 19440 & 16530 & 13080 \\
& 90 & 26050 & 38350 & 39250 & 35300 & 32030 & 26140 & 21390 & 17600 & 14570 \\
\hline
\end{tabular}

TABLE 3: Polarization degree and ratio with different concentrations.

\begin{tabular}{lccccccccc}
\hline \multirow{2}{*}{ Degree of polarization } & \multicolumn{8}{c}{ Concentration/g/L } \\
& 0.45 & 0.9 & 1.35 & 1.8 & 2.25 & 2.7 & 3.15 & 3.6 & 4.05 \\
\hline$P_{440}$ & 0.3303 & 0.3092 & 0.3146 & 0.3227 & 0.3222 & 0.3132 & 0.3053 & 0.3164 & 0.3031 \\
$P_{475}$ & 0.0354 & 0.0491 & 0.0426 & 0.0393 & 0.0359 & 0.0446 & 0.0478 & 0.0314 & 0.0539 \\
$P_{475} / P_{440}$ & 0.1072 & 0.1588 & 0.1354 & 0.1218 & 0.1114 & 0.1424 & 0.1566 & 0.0992 & 0.1778 \\
\hline
\end{tabular}

is reduced when the $\mathrm{pH}$ value is low, so the fluorescence intensity is decreased. When the $\mathrm{pH}$ value is larger than 3.88 , fluorescence quenching happens. It is because that the rise of alkalinity may cause long chain bending, which decreases fluorescence efficiency [21].

Compared with the polarized fluorescence spectrum in the condition of $0^{\circ}$, the spectrum in the condition of $90^{\circ}$ has a second peak. This result occurs mainly because when polarized fluorescence excites the fluorescent system, chromophores in sample solutions select light according to the excitation light polarization direction and the relative orientation of itself. In Table 2, when the polarization angle is $0^{\circ}$, $I_{440}$ is weaker than that of $90^{\circ}$. However, $I_{475}$ is stronger than that of $90^{\circ}$. Because the fluorescent molecules in the state of emission at $475 \mathrm{~nm}$ are more than fluorescent molecules in the state of emission at $440 \mathrm{~nm}$ with the polarization angle of $0^{\circ}$, similarly, fluorescent molecules in the state of emission at $475 \mathrm{~nm}$ are less than fluorescent molecules in the state of emission at $440 \mathrm{~nm}$ with the polarization angle of $90^{\circ}$. The more the fluorescent molecules in emission state are, the stronger the fluorescence intensity will be emitted. Otherwise, weaker fluorescence intensity will be emitted.

Table 2 exhibits the experimental data of fluorescence peak intensities of vinegar-water solutions detected with two polarization angles. Under the condition of $0^{\circ}, I_{457}$ is actually the superposition of $I_{440}$ and $I_{475}$ measured with polarization angle of $90^{\circ}$; then, $I_{440}$ and $I_{475}$ are chosen to perform analysis. In Table 2 , it is clearly observed that $I_{440}$ under the condition of $0^{\circ}$ is greater than that of $90^{\circ}$, but $I_{475}$ under the condition of $0^{\circ}$ is weaker than that of $90^{\circ}$.

From Table 3, polarization degree of each concentration is also calculated according to the theoretical calculation formula of fluorescence polarization:

$$
P=\frac{I_{\|}-I_{\perp}}{I_{\|}+I_{\perp}}
$$

$I_{\|}$is fluorescence polarization peak intensity of $0^{\circ} . I_{\perp}$ is fluorescence polarization peak intensity of $90^{\circ}$ [22]. Results are displayed in Table 3. $P_{440}$ is larger than $P_{475}$, and $P_{440}$ is $\sim 10$ times larger than $P_{475}$. Fluorescence polarization sensitivity is related to the collinear degree of excitation torque and emission torque. So intersection angle of absorption transition dipole torque and emission transition dipole torque under the irradiation at $440 \mathrm{~nm}$ is smaller than that under the irradiation at $475 \mathrm{~nm}$, which leads to a higher collinear degree and unobvious depolarization effect at $440 \mathrm{~nm}$ compared to that at $475 \mathrm{~nm}$.

\section{Conclusions}

After analysis and discussion, our conclusions are summarized as follows. (i) After being excited by UV light at $380 \mathrm{~nm}$, vinegar-water solutions with different concentrations emit florescence. The fluorescence emission peaks are at $445 \mathrm{~nm}$ and $470 \mathrm{~nm}$, and the emission type is $\mathrm{n} \rightarrow \pi^{*}$. (ii) The relationship of fluorescence intensity versus $\mathrm{CH}_{3} \mathrm{COOH}$ concentration follows Lambert Beer's law and fluorescence quenching effect. The relationship of fluorescence intensity versus $\mathrm{pH}$ values of vinegar-water solutions is related to the proton of the acid group, the coordination ability of the hydrogen bond, and the solubility or the situation of long chain. (iii) The relationship between the number/sensitivity of fluorescent chromophores and their collision probability decides the relationship between $I_{445}$ and $I_{470}$. (iv) For each concentration of vinegar-water solutions, the intersection angle of absorption transition dipole torque and emission transition dipole torque under the irradiation at $440 \mathrm{~nm}$ is smaller than that under the irradiation at $475 \mathrm{~nm}$. This result leads to a higher collinear degree and unobvious depolarization effect at $440 \mathrm{~nm}$ compared to that at $475 \mathrm{~nm}$. Hence, the report has a great significance in future studies about physical/chemical properties as well as cluster structures of vinegar.

\section{Competing Interests}

The authors declare that they have no competing interests. 


\section{Acknowledgments}

This research was founded by the National Natural Science Foundation of China (Grant no. 61575087), the Natural Science Foundation of Jiangsu Province (Grant no. BK20151164), and the Natural Science Foundation Project for Ph.D. Teachers of Jiangsu Normal University (Grant no. 9212214101). The authors would like to thank the project funded by the Priority Academic Program Development of Jiangsu Higher Education Institutions (PAPD) and Jiangsu Collaborative Innovation Center of Advanced Laser Technology and Emerging Industry for their generous financial support. The authors thank Lu Zhu and Whitney Ingram for proofreading the paper.

\section{References}

[1] Joint FAO/WHO Expert Committee on Food Additives. Food and Agriculture Organization of the United Nations, Compendium of Food Additive Specifications: Combined Specifications from 1st through the 37th Meetings, 1956-1990, Food and Agriculture Organization of the United Nations, Rome, Italy, 1992.

[2] Y. Yuan, F. Feng, L. Chen, Q. Yao, and K. Chen, "Directional isolation of ethanol-tolerant acetic acid bacteria from industrial fermented vinegar," European Food Research and Technology, vol. 236, no. 3, pp. 573-578, 2013.

[3] R. M. Callejón, J. M. Amigo, E. Pairo, S. Garmón, J. A. Ocaña, and M. L. Morales, "Classification of Sherry vinegars by combining multidimensional fluorescence, parafac and different classification approaches," Talanta, vol. 88, pp. 456-462, 2012.

[4] J. J. Wu, Y. K. Ma, F. F. Zhang, and F. S. Chen, "Biodiversity of yeasts, lactic acid bacteria and acetic acid bacteria in the fermentation of 'Shanxi aged vinegar', a traditional Chinese vinegar," Food Microbiology, vol. 30, no. 1, pp. 289-297, 2012.

[5] P. Wang, C. Y. Han, F. Y. Zhou, J. S. Lu, X. G. Han, and Z. W. Wang, "Electrochemical determination of tert-butylhydroquinone and butylated hydroxyanisole at choline functionalized film supported graphene interface," Sensors and Actuators BChemical, vol. 224, pp. 885-891, 2016.

[6] Z. Wang, F. Liu, and Y. He, "Comparison and determination of acetic acid of plum vinegar using visible/near infrared spectroscopy and multivariate calibration," in Proceedings of the WRI World Congress on Computer Science and Information Engineering, pp. 201-204, Los Angeles, Calif, USA, March-April 2009.

[7] J. Wu, X. Qin, C. Miao et al., "A honeycomb-cobweb inspired hierarchical core-shell structure design for electrospun silicon/carbon fibers as lithium-ion battery anodes," Carbon, vol. 98, pp. 582-591, 2016.

[8] E. Guzmán, V. Baeten, J. A. F. Pierna, and J. A. García-Mesa, "Evaluation of the overall quality of olive oil using fluorescence spectroscopy," Food Chemistry, vol. 173, pp. 927-934, 2015.

[9] T. Maki, N. Soh, K. Nakano, and T. Imato, "Flow injection fluorometric determination of ascorbic acid using perylenebisimide-linked nitroxide," Talanta, vol. 85, no. 4, pp. 1730-1733, 2011.

[10] H. Iizuka, K. Someya, and T. Yajima, "Hemin-mediated fluorometric determination of melatonin by high-performance liquid chromatography," International Congress Series, vol. 1304, pp. 409-414, 2007.

[11] P. Zhang, L. Liu, Y. Tao et al., "An amplitude modulation fluorometric method for phytoplankton classified measure," Optik, vol. 125, no. 11, pp. 2661-2664, 2014.
[12] Y. Zhao and X. Li, "Colorimetric and fluorometric detection of anionic surfactants with water soluble sensors," Sensors and Actuators B: Chemical, vol. 209, pp. 258-264, 2015.

[13] R. M. Silverstein, F. X. Webster, and D. J. Kiemle, Spectrometric Identification of Organic Compounds, Wiley, New York, NY, USA, 7th edition, 2005.

[14] C.-Q. Han, P.-T. Duan, Y. Liu et al., "Study on the fluorescence spectra characteristic of acetone-water solution," Laser Journal, vol. 31, no. 4, pp. 38-40, 2010.

[15] K. P. Huber and G. Herzberg, Molecular Spectra and Molecular Structure, Springer, New York, NY, USA, 1979.

[16] L. H. J. Lajunen and P. Peramaki, Spectrochemical Analysis by Atomic Absorption and Emission, The Royal Society of Chemistry, 2004.

[17] J. R. Lakowicz, Principles of Fluorescence Spectroscopy, Springer Science+Business Media LLC, 2006.

[18] R. L. Zhijun Wang and H. Lei, "Fluorescence spectral study on interaction of inositol and bovine serum albumin," Chemical Research, vol. 23, pp. 64-66, 2012.

[19] A. Kulmyrzaev and E. Dufour, "Determination of lactulose and furosine in milk using front-face fluorescence spectroscopy," $L e$ Lait, vol. 82, no. 6, pp. 725-735, 2002.

[20] R. Gillies, G. Zonios, R. R. Anderson, and N. Kollias, "Fluorescence excitation spectroscopy provides information about human skin in vivo," The Journal of Investigative Dermatology, vol. 115, no. 4, pp. 704-707, 2000.

[21] D. Chen, W.-Q. Liu, Y.-J. Zhang, J.-G. Liu, H.-B. Li, and Z.-Q. Ding, "Experimental investigation on the quenching characters of induced fluorescence of dissolved organic matter in polluted water," Spectroscopy and Spectral Analysis, vol. 26, no. 12, pp. 2283-2286, 2006.

[22] K. Itô, Encyclopedic Dictionary of Mathematics, MIT Press, 1993. 

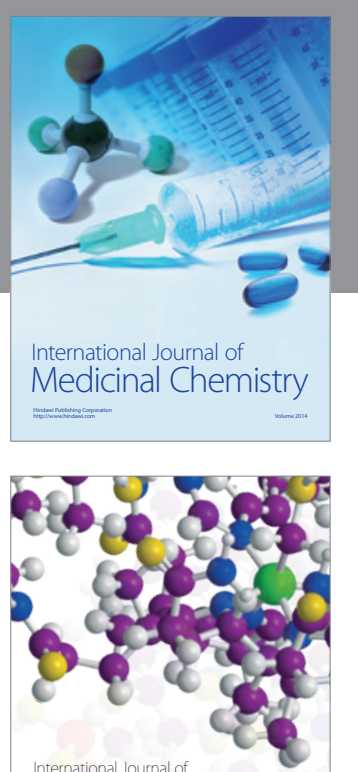

Carbohydrate Chemistry

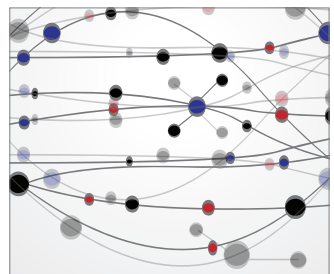

The Scientific World Journal
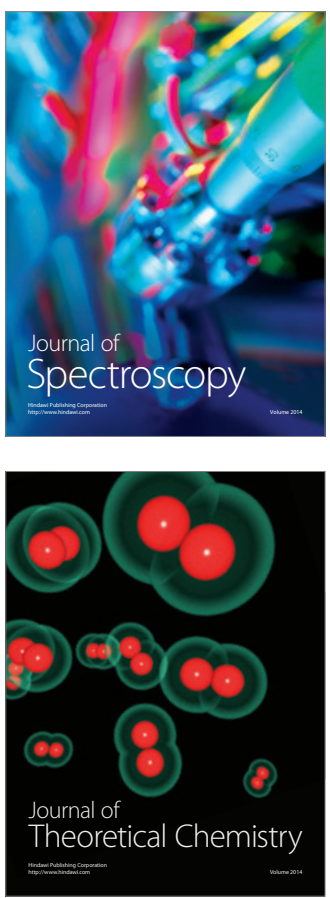
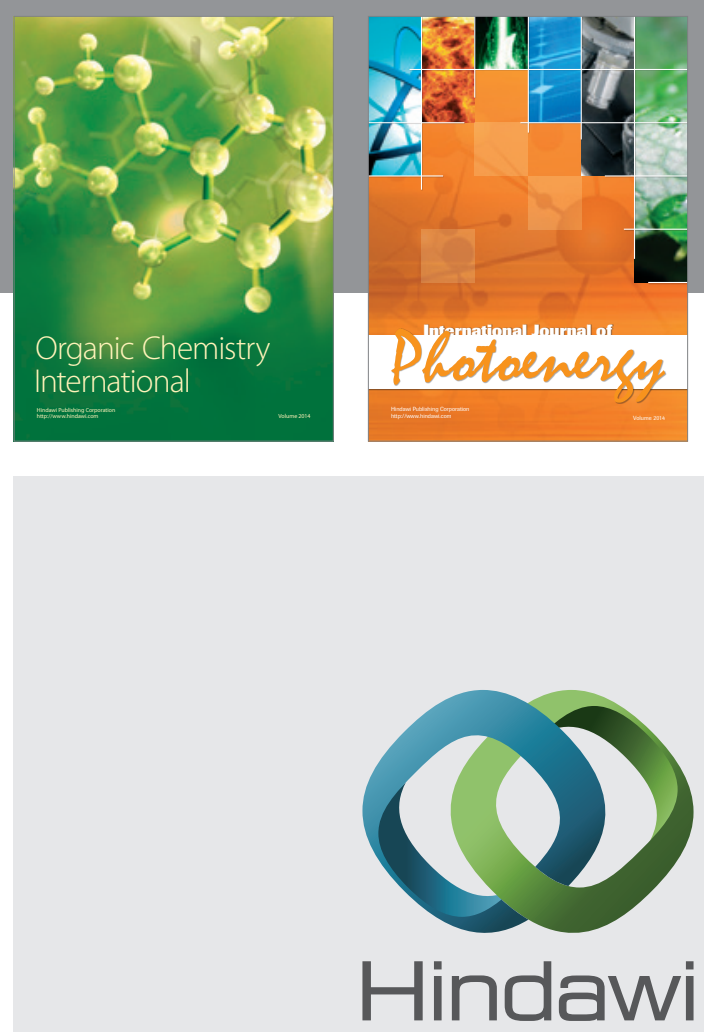

Submit your manuscripts at

http://www.hindawi.com

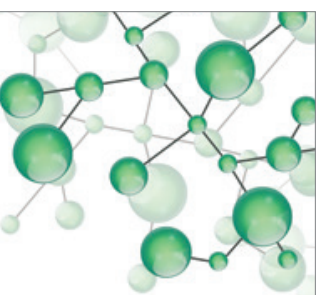

International Journal of

Inorganic Chemistry

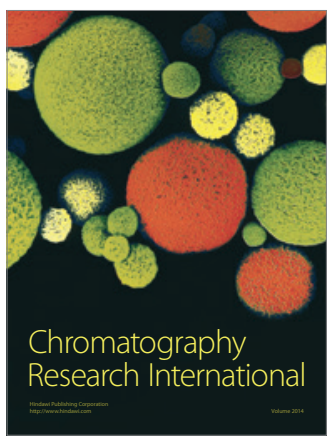

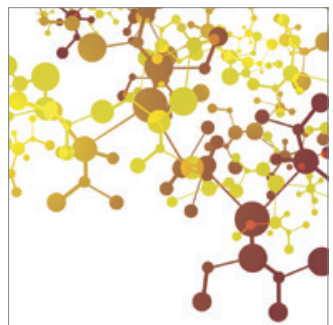

Applied Chemistry
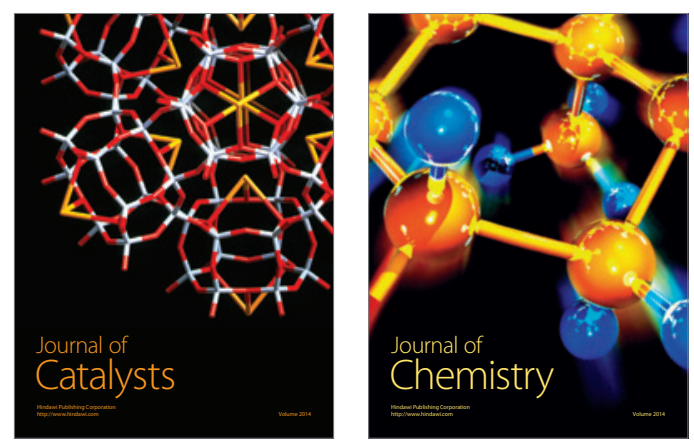
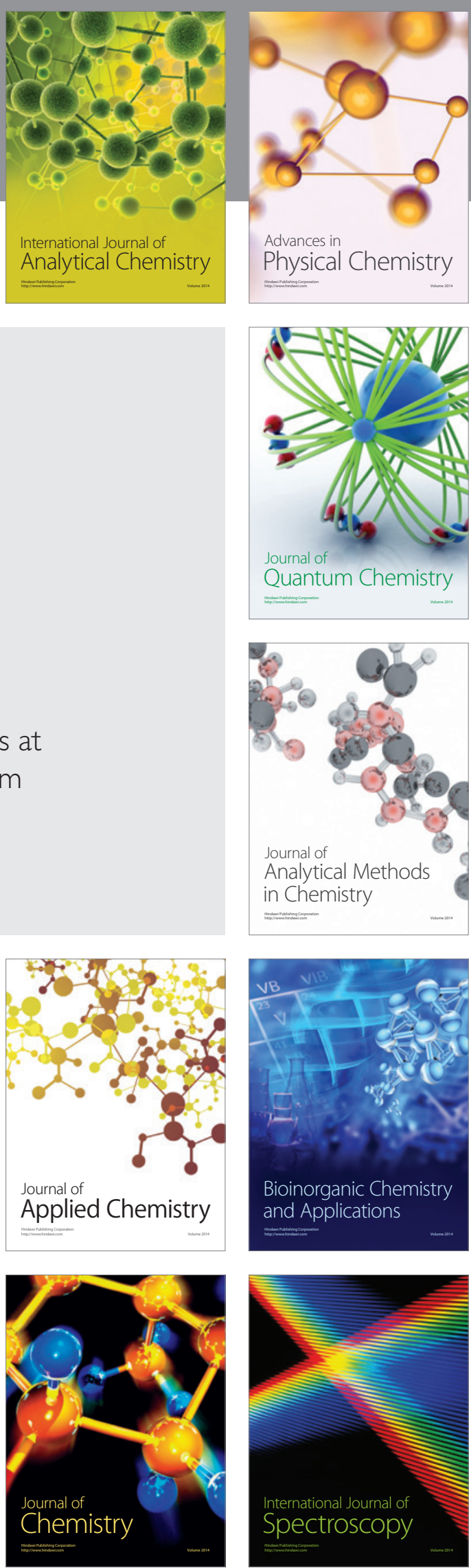